\title{
THE ABUNDANCES OF VEGA FROM THE ULTRAVIOLET SPECTRUM
}

\section{FIORELLA CASTELLI}

CNR-GNA c/o Osservatorio Astronomico

Via G.B. Tiepolo 11 - 34131 Trieste - Italy

\begin{abstract}
We derived preliminary abundances from the ultraviolet spectrum of Vega by comparing high-resolution Copernicus spectra with spectra computed with the ATLAS9 code and with updated line lists. The $T_{\text {eff }}=9400 \mathrm{~K}, \log g=3.90$ model, which fits the visual flux and the Balmer profiles is able to fit the ultraviolet flux either with $[\mathrm{M} / \mathrm{H}]=-1$ and microturbulent velocity $\xi=2 \mathrm{~km} \mathrm{~s}^{-1}$ or with $[\mathrm{M} / \mathrm{H}]=-0.5$ and $\xi=0 \mathrm{~km} \mathrm{~s}^{-1}$. The line spectrum is fitted by $\xi=2 \pm 0.5 \mathrm{~km} \mathrm{~s}^{-1}$ and by an iron abundance $\log \left(\mathrm{N}(\mathrm{Fe}) / \mathrm{N}_{t o t}\right)=-5.2 \pm 0.1$ dex. All the observed elements indicate underabundances ranging from about -0.35 dex (C) up to about -1 dex $(\mathrm{Ni}, \mathrm{Zn})$. The origin of the underabundances is an open question.
\end{abstract}

\section{INTRODUCTION}

Vega ( $\alpha$ Lyrae $=$ HR $7001=$ HD 172167) is a Pop. I star of spectral type A0 V with a projected rotational velocity $v$ sini $=23 \mathrm{~km} \mathrm{~s}^{-1}$ (Gray,1980). Abundances derived from the visual range are lower than the solar ones from about 0 to -0.9 dex (Venn and Lambert,1990; Adelman and Gulliver, 1990). Vega is not unique, but is part of a group of metal-mild-underabundant slow-rotating A-type stars of luminosity class III-V for which abundance analyses based on high-resolution observations have been done only recently (see Cowley,1991). The observed underabundances could be due to physical mechanisms acting either inside the stars, as the differential diffusion (Michaud,1980), or acting outside the stars in the interstellar medium, as the separation of interstellar grains and gas (Venn and Lambert,1990), or acting both inside and outside the stars (Charbonneau, 1991). A quite attractive explanation could be the selective accretion by only the gas forming toghether with dust a circumstellar disk (Waters et al.,1992). Infrared observations have shown the existence of such a disk around Vega.

We have been studying the ultraviolet spectrum of Vega in order to add information to the abundance pattern of this star which nearly always is chosen as comparison standard in studies of A-type stars.

\section{ANALYSIS AND RESULTS}

Preliminary abundances were derived by comparing in the 130-135 $\mathrm{nm}$ and 200$318 \mathrm{~nm}$ regions LTE computed spectra with Copernicus U1 and V1 spectra with 
resolutions of $0.005 \mathrm{~nm}$ and $0.01 \mathrm{~nm}$ respectively. The $U 1$ spectrum was observed by R.L. Kurucz in September 1974 and the V1 spectrum is published and exhaustively described by Rogerson (1989). Synthetic spectra were computed by using the SYNTHE code of Kurucz. The LTE model was computed by means of the ATLAS9 code with the new opacities (Kurucz,1991). The parameters $\mathrm{T}_{\text {eff }}=9400 \mathrm{~K}$ and $\log \mathrm{g}=3.90$ are obtained with the same methods and the same visual observed fluxes and observed Balmer profiles as decribed by Kurucz (1992). We derived the metallicity $[\mathrm{M} / \mathrm{H}]$ and the microturbulent velocity $\xi$ of the model from the comparison of the observed and computed ultraviolet fluxes. Observed fluxes are from IUE (Bohlin et al., 1990) and can be fitted either by a model with $[\mathrm{M} / \mathrm{H}]=-1$ and $\xi=2 \mathrm{~km} \mathrm{~s}^{-1}$ or by a model with $[\mathrm{M} / \mathrm{H}]=-0.5$ and $\xi=0 \mathrm{~km} \mathrm{~s}^{-1}$.

TABLE I Preliminary abundances of Vega from the ultraviolet spectrum.

$$
\log \epsilon=\log \left(\mathrm{N}_{\text {elem }} / \mathrm{N}_{\text {tot }}\right)
$$

\begin{tabular}{|c|c|c|c|c|c|c|}
\hline \multirow[t]{2}{*}{ Elem } & \multirow[t]{2}{*}{$\log \epsilon_{U V}$} & \multicolumn{2}{|c|}{$\log \epsilon_{V i s}$} & \multirow{2}{*}{$\begin{array}{l}\log \epsilon_{S \mathrm{un} 1} \\
\mathrm{AG} 1\end{array}$} & \multirow{2}{*}{$\begin{array}{c}\log \epsilon_{S u n 2} \\
G\end{array}$} & \multirow[t]{2}{*}{$\log \left(\epsilon_{U V}\right)-\log \left(\epsilon_{S_{u n} 1}\right)$} \\
\hline & & VL & AG & & & \\
\hline C I & -3.88 & -3.62 & -3.81 & -3.48 & -3.44 & -0.4 \\
\hline C II & -3.78 & & & -3.48 & -3.44 & -0.3 \\
\hline N I & -4.49 & -4.04 & & -3.99 & -4.04 & -0.5 \\
\hline O I & -3.71 & -3.30 & & -3.11 & -3.11 & -0.6 \\
\hline Mg I & -4.96 & & -5.11 & -4.46 & -4.46 & -0.5 \\
\hline Mg II & -4.96 & -5.33 & -5.15 & -4.46 & -4.46 & -0.5 \\
\hline Si I & $\leq-5.09$ & & & -4.49 & -4.49 & $\leq-0.6$ \\
\hline Si II & -5.09 & & & -4.49 & -4.49 & -0.6 \\
\hline Si III & -4.49 & & & -4.49 & -4.49 & 0 \\
\hline S I & $-4.83 /-5.83$ & -4.83 & & -4.83 & -4.77 & $0 /-1$ \\
\hline Ca II & -6.18 & -6.20 & & -5.68 & -5.70 & -0.5 \\
\hline Ti II & -7.80 & -7.49 & -7.47 & -7.05 & & -0.75 \\
\hline V II & -8.54 & & & -8.04 & & -0.5 \\
\hline Cr II & -7.12 & & -6.80 & -6.37 & & -0.5 \\
\hline Mn II & $>-7.15$ & & -7.24 & -6.65 & & $>-0.5$ \\
\hline Fe I & -5.2 & -5.06 & -5.05 & -4.37 & -4.52 & -0.83 \\
\hline Fe II & -5.2 & -5.19 & -5.16 & -4.37 & -4.52 & -0.83 \\
\hline Co II & $-8.12 ?$ & & & -7.12 & & $-1 ?$ \\
\hline Ni I & -6.79 & & -6.42 & -5.79 & & -1 \\
\hline $\mathrm{Ni}$ II & -6.79 & & -6.33 & -5.79 & & -1 \\
\hline Cu II & $-8.83 ?$ & & & -7.83 & & $-1 ?$ \\
\hline Zn II & -8.44 & & & -7.44 & & -1 \\
\hline
\end{tabular}


The computed line spectra are, however, independent from $\xi$ and $[\mathrm{M} / \mathrm{H}]$ adopted for computing the model, because both models (1) $\mathrm{T}_{e f f}=9400 \mathrm{~K}, \log \mathrm{g}=3.90$, $\xi=0 \mathrm{~km} \mathrm{~s}^{-1},[\mathrm{M} / \mathrm{H}]=-0.5$ and (2) $\mathrm{T}_{e f f}=9400 \mathrm{~K}, \log \mathrm{g}=3.90, \xi=2 \mathrm{~km} \mathrm{~s}{ }^{-1}$, $[\mathrm{M} / \mathrm{H}]=-1$ yield the same synthetic spectrum, provided that the same $\xi$ and the same abundances are used for computing the spectrum. The adopted line lists are basically those of Kurucz (1989).

The iron abundance $\log \left(\mathrm{N}(\mathrm{Fe}) / \mathrm{N}_{\text {tot }}\right)=-5.2 \pm 0.1$ dex was obtained from saturated strong Fe II lines, which weakly depend on $\xi$. After having fixed the iron abundance we derived $\xi=2 \pm 0.5 \mathrm{~km} \mathrm{~s}^{-1}$ from the other $\mathrm{Fe}$ II lines depending on $\xi$. Preliminary abundances results are summarized in Table I. They are compared with the abundances from the visual spectrum (Venn and Lambert,1990; Adelman and Gulliver,1990), and with the solar photospheric abundances (Anders and Grevesse,1989; Grevesse, 1991). All the elements are underabundant ranging from -0.35 dex for carbon up to -0.83 dex for iron and to $-1 \mathrm{dex}$ for $\mathrm{Ni}$ and $\mathrm{Zn}$. With the new solar iron determination of $-4.52 \mathrm{dex}$ the iron underabundance is reduced to -0.68 dex. Abundances of V, Mn, Co, and $\mathrm{Cu}$ may be affected by systematic errors, owing to the lack of hyperfine splitting in the calculations. Some lines, as, for instance, the blended features consisting mainly of $\mathrm{N} \mathrm{I}(12)$ at $131.8998,131.9005 \mathrm{~nm}, \mathrm{Mg}$ I (1) at $285.2127 \mathrm{~nm}$, S I (8) at $132.35156,132.3523 \mathrm{~nm}, \mathrm{Fe}$ II (1) at 259.837 and $259.9939 \mathrm{~nm}$, and the strongest lines of $\mathrm{Ti}$ II Vis. mult. 5 at $307 \mathrm{~nm}$ require solar or nearly solar abundances in order to be correctly reproduced.

\section{REFERENCES}

Adelman,S.J., and Gulliver,A.F. 1990, Ap.J., 348, 712.

Anders,E., and Grevesse, N. 1989, Geochim. Cosmochim. Acta 53, 197.

Bohlin,R.C.,Harris,A.W.,Holm,A.V.,Gry,C. 1990,Ap.J. Suppl.,73, 413.

Charbonneau,P. 1991, Ap. J. (Letters), 372, L33.

Cowley,C.R. 1991, IAU Symp. No 145, p.183.

Gray,D.F. 1980, Pub. A. S. P, $92,154$.

Grevesse,N. 1991, quoted as private communication by Iglesias C.A.,Rogers, F.J.,and Wilson,B.G. in Spin-Orbit Interaction Effects on the Rosseland Mean Opacity, 1992,Ap.J., preprint.

Kurucz,R.L. 1989, line lists on magnetic tapes

Kurucz,R.L. 1991, New Opacity Calculations in Stellar Atmospheres beyond classical models, ed. Crivellari et al., NATO ASI Series, p. 441.

Kurucz,R.L. 1992, New atmospheres for modeling binaries and disks, to be publised in Improvements in Light Curve Modeling of Eclipsing Binary Stars, Berlin,Springer-Verlag.

Michaud,G. 1980, Astron. J., 85, 589.

Rogerson Jr.J.B. 1989, Ap. J. Suppl., 71, 1011.

Venn,K.A., and Lambert,D.L. 1990, Ap.J.,363,234.

Waters,L.B.F.M.,Trams,N.R., Waelkens,C. 1992, Astr. Ap., preprint. 